\title{
Genetix
}

Take the lead

\section{Rapid selection of mammalian cells secreting large amounts of therapeutic proteins or peptides}

Screening cell populations for high producing clones is a key rate-limiting step in the production of protein therapeutics. The Genetix ClonePix FL provides a powerful way to find and collect the highest producing clones in a rapid one-step process. Clonal colonies of transfected CHO, HEK293 or myeloma cells secreting a recombinant protein of interest are probed in situ with target protein-specific, fluorophoreconjugated antibody, and then selected based on their protein secretion rate using robotic processing and picking techniques. This process considerably improves efficiency and streamlines traditional workflows, generating optimal cell lines that secrete the highest levels of the desired protein or peptide.

The ClonePix FL system works by imaging thousands of producer cells grown into discrete colonies (either suspended in or grown adherently under semisolid medium) using fluorescence specific to the protein or peptide of interest. These images are then analyzed to select the colonies that have the highest fluorescence for a given colony volume (that is, the highest producers of the protein of interest). Up to five fluorescence wavelengths can be multiplexed with brightfield images, thus allowing multiple probes to be used in a single picking run or to measure other parameters such as cell viability (using the Genetix LiveDetect reagent).

\section{The ClonePix FL selection process}

After transfecting the cell line with the construct encoding the protein of interest, the cells are grown under selection using standard methods and plated into CloneMatrix-based semisolid medium. The semisolid medium can be made up using a $2 \times$ concentrate of the medium of choice. Cell density should be determined empirically for each cell line, but guideline densities are 200 cells/ml for $\mathrm{CHO}$ cells and 1,000 cells/ml for HEK293 cells. Both serum-containing and chemically defined expression media can be used.

The fluorescently conjugated capture and detection antibodies are either added to the medium at the time of plating or sprayed on using an atomizer at least $24 \mathrm{~h}$ before imaging and picking the colonies. In either case, recommended final antibody concentration is $7-10 \mu \mathrm{g} / \mathrm{ml}$, although it may be possible to titrate particular antibodies to lower concentrations or increase antibody concentration to improve image intensity.

\section{Edmund Newman \& Christopher J Mann}

Genetix, Queensway, New Milton, Hampshire BH25 5NN, UK. Correspondence should be addressed to E.N. (edmund.newman@genetix.com).

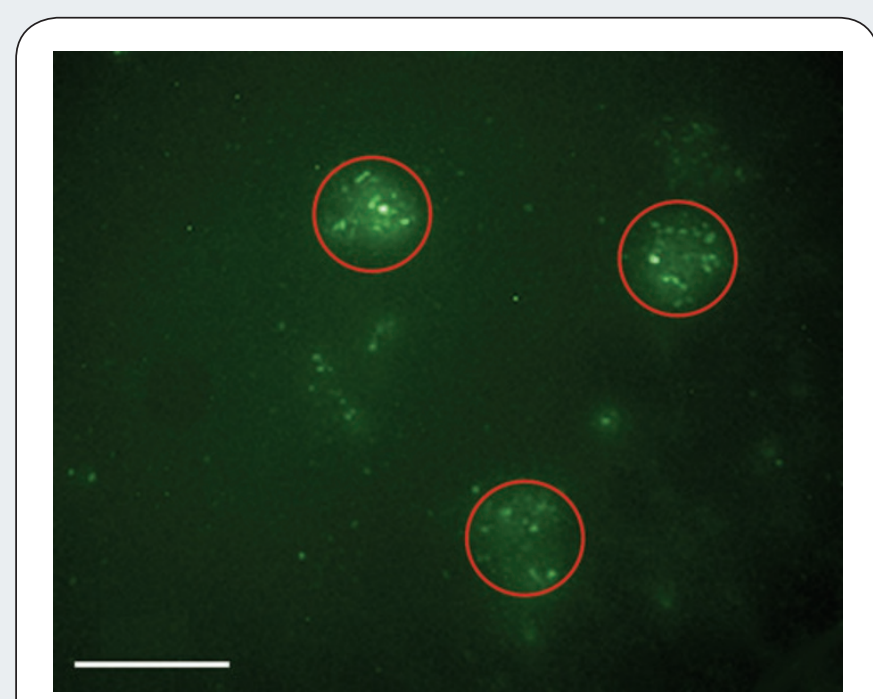

Figure 1 | Detection of high-secreting colonies of HEK293 cells stably transfected with an untagged monomeric protein and grown under CloneMatrix-based semisolid medium. Detected with fluorescein isothiocyanate (FITC)-conjugated antibody specific to the protein. Image is colorized in green, and high secretors are circled in red. Scale bar, $1 \mathrm{~mm}$.

Cells can be grown either as adherent colonies in tissue culturetreated plates or as suspension colonies in non-tissue culture-treated plates. It is preferable to grow producer cells as suspension colonies wherever possible, as this improves image quality and picking efficiency. Adherent cells, such as CHO-K1 and HEK293, can be grown as suspension colonies in semisolid medium.

After the cells have grown into colonies of about 100 cells (typically after 7-12 d), the plates are fluorescently imaged using ClonePix FL. Genetix PetriWell 6-well and 1-well plates have been designed 
specifically for this application to minimize the autofluorescence and flaring that occurs with regular culture plates. The ClonePix FL software then automatically analyzes fluorescence intensity (and therefore productivity) and picks the highest-secreting colonies into separate

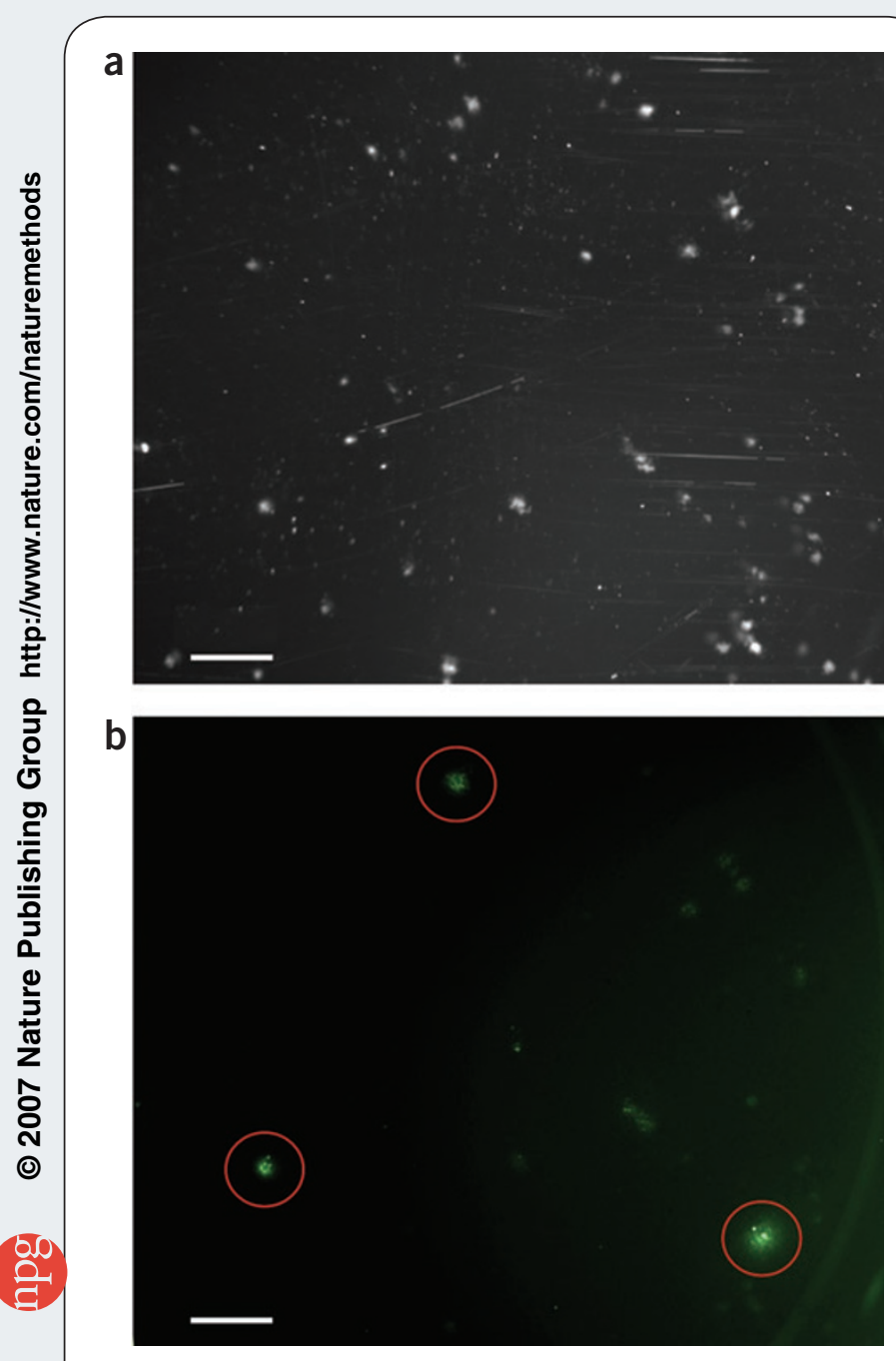

Figure $\mathbf{2}$ | Detection of high-secreting colonies of adherent $\mathrm{CHO}$ cells stably transfected with a monomeric protein construct containing both His and Flag tags at the $\mathrm{C}$ terminus of the protein, and grown in suspension in CloneMatrixbased semisolid medium. (a) Brightfield image showing cell colonies.

(b) Detection of secreted protein using a combination of anti-His and anti-Flag, of which the anti-His was conjugated with FITC. The image in $\mathbf{b}$ is colorized in green, and high secretors are circled in red. Scale bars, $1 \mathrm{~mm}$. wells of a 96-well destination plate containing the medium of choice for outgrowth and scale-up. The resulting cell lines are clonal, highproducer cell lines.

\section{Selection of cells producing untagged protein}

If the protein of interest is untagged, for example an endogenous or wild-type transfected protein, its secretion can be detected and quantified using a fluorescently conjugated antibody specific to that protein (Fig. 1). For the detection complex to form correctly, it is important to use a polyclonal antibody or several monoclonal antibodies raised to different epitopes of the protein.

\section{Selection of cells producing peptide-tagged protein}

When it is not possible to detect the protein of interest directly because a specific antibody is not available, or if a common detection system is preferred, then recombinant proteins can be detected via peptide tags such as c-myc or Fc using a capture antibody to the tag. Where short amino acid tags are used (for example His, HA, Flag and others) it is important that the constructs encode two epitope tags to achieve efficient formation of the detection complex. These can be either a tandem repeat of the same tag or two separate tag sequences, and can be added to the protein sequence in tandem at one terminus or at opposite termini. When using two different epitope tags, the detection antibodies should be applied in molar equivalence. Figure 2 shows an example of a secreted protein detected via tandem C-terminal His and Flag tags.

\section{Conclusions}

Using ClonePix FL technology removes the need for serial dilution or cell-sorting techniques. It allows monoclonal populations of highsecreting cells to be established in a much shorter time frame, removing many of the bottlenecks associated with producer-cell-line optimization and downstream scale-up for protein or peptide production. The low labor requirement means that more cell populations can be interrogated in parallel, increasing cell-line throughput. It is estimated that one ClonePix FL used by two scientists is sufficient to take more than 100 cell populations from transfection to the shake-flask stage per year.

This article was submitted to Nature Methods by a commercial organization and has not been peer reviewed. Nature Methods takes no responsibility for the accuracy or otherwise of the information provided. 